\title{
Collaborative Filtering Algorithm Based on Social Network Information Flow Model
}

\author{
Mingwei Sun ${ }^{1, a}$, Qiang Liu² and Enyang $\mathrm{Gao}^{3^{*}}$ \\ ${ }^{1}$ Tonghua Normal University. Tonghua; China; \\ 2 Jilin Engineering Normal University .Changchun; China; \\ ${ }^{3}$ Shenyang Jianzhu University, Shenyang, China \\ a2238552865@qq.com \\ *The corresponding author
}

Keywords: Communication technology; Collaborative filtering; Multidimensional half; Semi markov process

\begin{abstract}
In order to improve the accuracy of personalized recommendation technology, the state space of multidimensional semi markov process definition "empty state" has been extended to multidimensional markov process, combining with the social network analysis theory and social network information flow model. The model describes the process flow of information in members of the network society. Then, based on social network information flow model, collaborative filtering is put forward to SMRR. By comprehensive considering user's preferences and clubs, the influence of network of other members SMRR prediction accuracy is significantly higher than the original algorithm.
\end{abstract}

\section{Introduction}

Collaborative filtering algorithm is studied by the user's individualized demand. Actively recommend to the user is most likely to accept resources (products, technology or view), or find most likely to choose some kind of resource use. The traditional collaborative filtering algorithms assume that similar users to select similar resources through calculating the user. Source of most similar to each user is recommended. However, For the choice of a resource, it has been exchanged in the direct of information users mutual influence, which is more than the similarity between users, direct and effective $[1,2,3]$.

In the electronic commerce application, system data record contains user. The interaction between relationships is achieved, such as user calls relationship of communication between the networks because people are social activities and formation of the network as the social complexes network.

In this article, innovation diffusion theory to a new technology or new ideas in the society has been proposed. In the network, it is accepted gradually by the members of the process modeling. As social members, Rogers can be divided into two groups of pioneers and fellow travelers. Bass model through judgment than a pioneer and a follower is built; the transmission range is quantitative innovation in social network. But the bass model ignores the influence of social network structure on information diffusion because of considering the information of diffusion velocity in the network. According to the influence of the interaction relationship between users of the user model, this kind of influence on the spread of the social network has been found in the research. How to maximize innovation under the limited consumption is the valuable problem. Continuous time markov chain is established based on the diffusion rate of information flow model, and is applied to collaborative filtering algorithm. But members of the interaction between interval must obey exponential distribution, it's not only limiting the generality of the model, but do not tally with the actual research. The information flow model only considers the single clubwill the mutual influence between members, without taking into account the social members belong to the network of substructure (community structure) between the user information transmission probabilities. 
According to user's position in the network The influence is given $[4,5,6]$.

\section{Social Network Information Flow Model}

The Information Flow Model Based On Extension Half A Markov Process. Traditional semi markov process state space $\mathrm{S}$ for one dimensional empty in the state space can be extended to a multi-dimensional space ( $m$ dimensionality),

In the half markov process, the random variable $X \mathrm{n}=\{X 1, X 2, \ldots, \mathrm{m}\}$ for $\times$ Space $S * S * m=$ S... X, within the random vector, including $S=\{I 0,1, \mathrm{I} \ldots \mathrm{I} \mathrm{k}\}$. In practice, however, a class of process which is consisted of eight members of the social network has to been the member of edge represents the source point with a certain probability to point intended to convey information. Only I postulate that $\mathrm{T} 1$ social network 1 and $2 \mathrm{I}$ to the recipient.

Definition 1 (extended multidimensional semi markov process) $X=\{X n=\langle 1 \mathrm{x}, 2 \mathrm{x}, \ldots, \mathrm{x} \mathrm{m}\rangle$ n0 or higher, 1 or lessM $<$ up $\}$, Definition 1 (extended multidimensional semi markov process) $X=$ $\{\mathrm{X} \mathrm{n}=<1 \mathrm{x}, 2 \mathrm{x}, \ldots, \mathrm{x} \mathrm{m}>\mathrm{n} 0$ or higher, 1 or less $\mathrm{M}<\mathrm{up}\}$, the fixedn0 or higher, $\mathrm{X} \mathrm{n}$ is valued in state space $S \mathrm{~m}=$ Fig.

Process of information flow in social network, GS * $\mathrm{S} * \ldots$ wu s.m The random variables, including $\mathrm{S}=\{\mathrm{e}, \mathrm{I} 2,3, \mathrm{I} \ldots, \mathrm{I} \mathrm{k}\}$ when $\mathrm{X} \mathrm{X} \mathrm{I}=\mathrm{e} \mathrm{n}$, the random variable $\mathrm{X}$ (I) and the other dimension Degree of random variables are independent of each other, otherwise interconnected, state was According to the process $\{X . N=\{T\}(X, T, n), n 0$ or higher $\}$ is the extended multidimensional half horse, a markov process. This paper will extend multidimensional semi markov process for social networks by analysising social network information flow model.

Definition 2 (social network information flow model) $\mathrm{G}$ social network $=\{\} \mathrm{V}, \mathrm{E}, \mathrm{V}$ is the set of all nodes, $\mathrm{V} \mathrm{I}, \mathrm{V} \in$ j.teraction relationship between $\mathrm{V} \mathrm{V}=\mathrm{N}, \mathrm{j}(\mathrm{VI}, \mathrm{V})$ E.A random vector $\mathrm{X}=\{\mathrm{X}$ $\mathrm{n}=\langle 1 \mathrm{X}, 2 \mathrm{X}, \ldots \mathrm{N}, \mathrm{x}>, \mathrm{N} 0$ or higher $\}, \mathrm{X} \mathrm{n}$ is valued in state space $\mathrm{S}, \mathrm{n}=\mathrm{S} *(\mathrm{~S}-+\{\mathrm{e}\}\{1\} \mathrm{x}) \mathrm{x} \ldots$ $X(S-\{x\} N-1+\{e\}) N$ Random variables, namely $X n$.Each are not identical, the $S=\{e, I, 1,2$, $\mathrm{I}$..., I N \}, e is empty, $\mathrm{J} \mathrm{I} \mathrm{j}=\mathrm{g}(\mathrm{v}), \mathrm{I} \mathrm{j} \in \mathrm{S}, \mathrm{v}, \mathrm{j} \in \mathrm{vg} \mathrm{v}$ for the space to $\mathrm{S}-\{$ e\}Bijection. Value of $\mathrm{X} n$ said $\mathrm{T} n$ time, receives the information Households. $\mathrm{T}=\{\mathrm{T}, \mathrm{n}, \mathrm{n} \mathrm{p} 0\}, \mathrm{T}$ value $\mathrm{n}$ is nonnegative random variables, and $0=\mathrm{T} \mathrm{T} 10$ or less or less... T $\mathrm{n}$ or less... . $\{\mathrm{X}, \mathrm{T}\}=$ if process $\{(\mathrm{n}, \mathrm{XT}, \mathrm{n}), \mathrm{n} 0$ or higher $\}$ is a multidimensional half's extension process, said Cheng $\{X, T\} G$ network information flow model for the society. Social network flow model is extended multidimensional semi markov process. In the special case of this extension multidimensional random vector of semi markov process, each dimension value is the same. In addition to the empty state, each state value only one corresponds to the social network model of a node. In this condition, to a certain probability to become the recipient, in the next period of time can open. Messages are sent to other members of the beginning of give information to other members. Distribution is the social network interaction time interval between the two members in the league. The distribution function can be built in any form. In the study, Wen Offer[7] has found that the social interaction between the nodes in the network time Interval distribution obey the power law distribution, namely, $F(t)=t$ - beta, beta is constant (1)Set $P\{X n+1=$ alpha j, T $n+1-T n T \mid X$ or less $n=$ alpha $I\}=Q$ (alpha I, alpha j, t), the alpha I, alpha $\mathrm{j} \in \mathrm{S} \mathrm{m}$ for information flow in the model

The Influence of Social Network Structure of Transition Probability. The edge of social network figure structure of neutron density reflects the sub graph structure of the close degree of communication between nodes. General edge density is big and existed the higher the probability of mutual information between nodes. In this paper, community structure of links between nodes is close degree in the community. Edge density is greater than the edge density between communities [8]. In this paper, literature [9] has proposed algorithm $\mathrm{C}=$ social network will be divided into community structure $\{\mathrm{C} 0,1, \mathrm{C} \ldots, \mathrm{n}\} \mathrm{C}, \mathrm{C}$ in the form of a matrix, where Cij community $\mathrm{I}$ and $\mathrm{j}$ between edge article number. 


\section{SMRR Collaborative Filtering Algorithm}

Computing Information First time. Information flow model of each state first time matrix M, the yuan prime $\mathrm{m}_{\mathrm{ij}}$ for the first time for initial state, is I to live up to the expectations of $\mathrm{j}$. Make $\mathrm{R}=$ limt- > $0 \mathrm{Q}(\mathrm{I}, \mathrm{j}, \mathrm{t})$, the elements in the matrix R RijIn half a markov The rate at which range from state I to j. Take a constant $v$ Max or(R I) to replace $r$ of the diagonal elements to $1-r \mathrm{I} / \mathrm{v}$, so as to offer. To take out the markov chains with continuous time markov chain equivalent discrete. First of discrete markov chain time matrix is calculated and then converted to connect.

User Potential Value. The value of the user in addition to their own choice of some resources. The influence to other users is also the important measure. In this paper. Send messages to other users to a single user abstraction are the process of semi markov process, process state space $\mathrm{S}=$ $\{\mathrm{I} 1,2, \mathrm{I} \ldots, \mathrm{I} \mathrm{N}\}, \mathrm{I} \mathrm{j}$ and one to one correspondence of a member of a social network, $0=\mathrm{T} 0$ or less $\mathrm{T} 1$ or less... $\mathrm{T} \mathrm{n}$ or less... For the time series of state transition.

SMRR Algorithm. Set $(\mathrm{X}, \mathrm{T})$ is a single user to select resources in social networks Semi markov process, the current moment for $\mathrm{T} \mathrm{n}, \mathrm{Ti}$. $\mathrm{N}+1$ for the user to the I preferences of the next resource selection. SMRR algorithm defined in: A user's preferences of resource selection time and its average first time $\mathrm{m}$ j-The smaller the difference, the possibility of a more practical choice resources Big. In order to make more users accept resources in social networks.

\section{Conclusion}

Based on the social network information flow model of collaborative filtering algorithm SMRR, the concept of "empty state" as a multidimensional and half horse's process is introduce, combining with the feature of social network structure of social networks is put forward to information flow model. Partial SMRR algorithm is considered that the user itself is a good choice cycle and other users of social networks on their choice weeks. The influence of period has been improved to the prediction accuracy. At the same time, using paid half horse's model, according to the structure features and user's social network with neighboring user information interactive dynamic characteristics determine the user's potential value, making the suggested users can cause more social networks to select the resource. Finally, SMRR is finding that it is an accuracy algorithm. The original algorithm has obviously improved, and the recommended users more potential value.

\section{References}

[1] Chun-xiao Xing, Gao Fengrong, etc. To adapt to the change of user interest Collaborative filtering recommendation algorithm [J]. Journal of computer research and development, 2007, 44(2):296-301.

[2] Xing,Chun xiao, Gao Feng rong, Zhan Si - nan, et al. A collaborative filtering recommendation algo - Rithm incorporated (nasdaq: PCLN - news) with user interest change [J].Journal of Computer Research and Development, 2007, 44 (2): 296-301.

[3] SongXiao Dan, Chi Yun, Mr Hino Koji, et al. Infor -Mation flow modeling -based on coursing together what forPrediction and ranking // [C] Proceedings of the Six -Teenth International World Wide Web Conference, Banff, Alberta, Canada, 2007:191-200.

[4] kemper D, Mr. Kleinberg J, Tardos E.M aximizing the spread of influence through a social network [C] //Proc of 9 th ACM SIGKDD, Washington, DC, USA, 2003:301-311.

[5] Sarwar B, Karypis G, Konstan J, et al. The Item - -based Collaboratovefiltering recommendation algorithms[C] // Proceedings of the Tenth International World Wide Web Conference, Hong Kong, China, 2001:285-295.

[6] Bass F.A new product growth for the model of consumer Durables [J]. Journal of Management Science, 1969, 15 (5):215-227. 
[7] Xiaofu Ma, Yu Fang, Xingzhen Bai, "A Balanced Energy Consumption Clustering Algorithm for Heterogeneous Energy Wireless Sensor Networks", IEEE International Conference on Wireless Communications, Networking and Information Security, 2010:382-386.

[8] Xiaofu Ma, Haris I. Volos, Xiangwei Zheng, Jeffrey H. Reed, and Tamal Bose, "A Variation-Aware Approach for Task Allocation in Wireless Distributed Computing Systems", IEEE Global Communications Conference, 2013:5006-5011.

[9] Oliveira J. G., Barabasi A. L. Human dynamics: Dar -Win the and Einstein correspondence patterns [J]. Journal of $\mathrm{Na}$-True, 2005, 4(37):45-47. 